\title{
Les rives lacustres comme espaces publics : essai de cartographie. L'exemple du lac d'Annecy
}

Lakeshores as public spaces: a cartographic essay. The case of Lake Annecy Las riberas lacustres como espacio público: ensayo cartográfico a partir del ejemplo del lago de Annecy

\section{Alice Nikolli}

\section{OpenEdition Journals}

Édition électronique

URL : http://journals.openedition.org/mappemonde/407

DOI : 10.4000/mappemonde.407

ISSN : 1769-7298

\section{Éditeur}

UMR ESPACE

\section{Référence électronique}

Alice Nikolli, « Les rives lacustres comme espaces publics : essai de cartographie. L'exemple du lac d'Annecy », Mappemonde [En ligne], 123 | 2018, mis en ligne le 14 février 2018, consulté le 20 juin 2019 URL : http://journals.openedition.org/mappemonde/407 ; DOI : 10.4000/mappemonde.407

Ce document a été généré automatiquement le 20 juin 2019.

\section{cc) (†) (2)}

La revue Mappemonde est mise à disposition selon les termes de la Licence Creative Commons Attribution - Pas d'Utilisation Commerciale - Partage dans les Mêmes Conditions 4.0 International. 


\section{Les rives lacustres comme espaces publics : essai de cartographie. L'exemple du lac d'Annecy}

Lakeshores as public spaces: a cartographic essay. The case of Lake Annecy Las riberas lacustres como espacio público: ensayo cartográfico a partir del ejemplo del lago de Annecy

\section{Alice Nikolli}

1 Les espaces publics font l'objet d'une vaste littérature en sciences sociales depuis les années 1970, qui les considèrent comme des lieux de vivre-ensemble permettant d'assurer la coprésence entre les individus (Ghorra-Gobin, 2001; Lussault, 2003; Berdoulay et al., 2004). La notion d'espace public dépasse la seule dimension de la propriété foncière et désigne un « espace matériel aménagé à des fins d'utilisation par la collectivité [...], reconnu comme étant praticable et librement accessible à tout un chacun » (Dessouroux, 2003). Les espaces publics ne sont donc pas réductibles aux espaces de propriété publique, dans la mesure où des espaces de propriété privée peuvent être considérés comme des espaces publics, au sens d'espaces accessibles au public, tandis que des espaces de propriété publique peuvent fonctionner avec un accès réservé, voire privatisé.

2 Si les sciences sociales se sont longtemps concentrées sur les espaces publics urbains (places, rues, squares...), nombre de travaux s'intéressent désormais à « l'expansion » des espaces publics hors de la ville (Tomas, 2001) et étudient les «espaces ouverts» des franges urbaines (Delbaere, 2010), les « espaces publics de nature " (Clarimont, Leichnig, 2014), ou encore les espaces forestiers, la montagne, les rives des cours d'eau et des lacs (Tomas, 2001). Cette diversification des espaces publics tient notamment au développement des pratiques récréatives de nature, qui participent d'une nouvelle habitabilité des lieux géographiques (Bourdeau et al., 2011). Or, de manière plus symptomatique que les espaces publics urbains, ces espaces publics hors la ville ne sont pas nécessairement de propriété publique (Sontag, Roux, 2013). Les pratiques récréatives 
de nature portent en effet aussi bien sur des espaces de propriété publique (domaine public maritime, domaine public fluvial...) que de propriété privée (alpages, espaces agricoles périurbains...). Cela pose la question de l'articulation, et d'une potentielle déconnexion, entre le statut de propriété du sol et la régulation concrète de l'accès à ces espaces.

Cet article se propose d'étudier les rives du lac d'Annecy (Haute-Savoie, France) comme un de ces espaces publics «en expansion » qui posent la question des relations entre propriété foncière et accès à l'espace, en lien avec le développement des pratiques récréatives de nature. Ce lac est en effet très fréquenté pour des loisirs diversifiés (flânerie, sports nautiques motorisés ou non, baignade...) et fait l'objet d'une forte demande d'accès de la part des habitants et des touristes. Pour autant, le statut d'espace public des rives du lac ne va pas de soi. Si le plan d'eau en lui-même relève bien du domaine public fluvial, ce n'est pas le cas de ses rives, qui font l'objet de formes d'appropriation concurrentes (certaines publiques, d'autres privatives). La répartition entre espaces publics et espaces privatifs suscite ainsi des débats, voire des conflits autour de ce lac, notamment à l'occasion de projets d'aménagement ou d'évolutions législatives. Les rives du lac d'Annecy, comme celles d'autres grands lacs alpins, apparaissent ainsi comme des espaces convoités, support d'usages multiples et objet de volontés antagonistes d'appropriation.

4 L'article propose une réflexion sur les relations entre propriété foncière et accès concret dans la pratique des espaces récréatifs, fondée sur une analyse cartographique visant à explorer la déconnexion entre ces deux données et les articulations qu'elle génère. Après être revenu sur l'état des travaux géographiques concernant l'accès aux espaces récréatifs, l'article présente le terrain d'étude et la méthodologie mise en place pour la cartographie de la propriété et de l'accès ${ }^{1}$. Il croise ensuite les résultats de ces deux cartographies et met en lumière des enjeux de partage de l'espace le long d'un littoral convoité.

\section{L'accès aux rives lacustres pour les pratiques récréatives : état de l'art}

\section{Les littoraux lacustres, grands oubliés de la littérature sur l'accès aux espaces récréatifs?}

5 L'accès et les usages des espaces récréatifs ont fait l'objet de nombreux travaux, qui portent sur des types d'espaces diversifiés. Tout un pan de cette littérature s'intéresse aux espaces ruraux et périurbains (Mermet, Moquay, 2002; Michel, 2003; Le Caro, Kergreis, 2007), avec une approche centrée sur les conflits d'usage induits par le développement des pratiques récréatives, qui entrent en tension avec des usages traditionnels (agriculture, chasse, etc.). Les rivages maritimes constituent un autre pan de cette littérature, que l'on pense aux travaux sur les sentiers littoraux (Meur-Férec, 2007 ; Melin, 2015), sur l'occupation informelle du rivage camarguais sous forme de cabanes (Nicolas, 2008), sur la conflictualité liée à la diversité des usages littoraux (Cadoret, 2011) ou encore sur les espaces publics littoraux en milieu urbain (Fleury, 2005 ; Barthel, 2006). En revanche, peu de travaux portent spécifiquement sur l'accès à ces espaces littoraux, entendu comme possibilité concrète "de pénétrer et de circuler sur un espace » (Michel, 2003) et étudié du point de vue de sa potentielle restriction, et notamment de sa 
privatisation. Dans sa thèse portant sur le littoral en Océanie, Olivier Hoffer (2013) fait le constat que cet accès stricto sensu constitue un " terrain peu défriché par les géographes ", qui contraste avec la «profondeur insondable» des travaux consacrés aux littoraux en général.

6 Les littoraux lacustres font figure de grands absents de cette littérature sur les accès et les usages des espaces récréatifs. D'une manière générale, les lacs constituent un angle mort de la géographie humaine, à la différence d'autres territoires de l'eau, comme les zones humides (Sajaloli, 1996 ; Amalric, 2005) ou les fronts d'eau urbains maritimes ou fluviaux (Gravari-Barbas, 1991 ; Gérardot, 2007 ; Comby, 2013). La géographie des lacs (Touchart, 2000) est en effet une géographie purement physique, qui passe sous silence la question des usages lacustres, à l'exception régionale des travaux de Jean-Claude Vernex sur les lacs savoyards (Vernex, 1985 et suiv.). Plus récemment, les grands lacs alpins ont fait l'objet de plusieurs programmes de recherche pluridisciplinaires croisant les aspects physiques et sociaux (Magni, Chinaglia, 2007; Mori et al., 2007 ; Montuelle, Clemens, 2015). Dans ce contexte, l'accès aux littoraux lacustres est évoqué ponctuellement, en particulier face aux formes d'appropriation privative de l'espace (Vernex, 1985 ; Mori et al., 2007 ; Montuelle, Clemens, 2015), mais il n'est jamais l'objet principal des travaux en question. En anglais, on repère quelques publications sur l'accès aux littoraux lacustres, mais qui restent très isolées (Orr, Pickens, 2003 ; Hrafnkelsdóttir, 2010).

Cette rareté des travaux concernant l'accès aux grands lacs contraste avec l'acuité toute particulière que prend cette question dans un contexte où les pressions touristiques, urbaines et foncières sont fortes. Si ces pressions ont été mieux étudiées à propos des littoraux maritimes (Buhot et al., 2009), elles apparaissent sinon égales, du moins comparables, sur les littoraux de certains grands lacs alpins très urbanisés et mis en tourisme, qu'il s'agisse du Léman, du lac d'Annecy ou encore des grands lacs italiens (Magni, Chinaglia, 2007 ; Laslaz et al., 2015).

\section{L'accès à l'espace, entre " privatisation » et " publicisation »}

8 L'accès aux espaces récréatifs est pris dans une dialectique qui articule dynamiques de privatisation et de publicisation. La privatisation constitue un questionnement récurrent dans la littérature relative aux espaces publics urbains. Ces travaux insistent sur la prolifération de territoires privatisés, qui annoncerait la disparition de tout espace public (Decroly et al., 2003). En cela, ces analyses s'inscrivent dans un courant de recherche qui emprunte à la géographie radicale anglo-saxonne et à sa critique de la ville néolibérale fragmentée et ségréguée (Davis, 1992 ; Mitchell, 2003). Ces travaux font ainsi le constat d'une «atomisation accrue des territoires urbains, desquels les espaces publics traditionnels [...] seraient en passe de disparaître au profit d'une constellation d'enclaves aisées exclusivement dévolues à la consommation ou à l'entre-soi de leurs usagers " (Decroly et al., 2003). Toute une littérature s'intéresse ainsi aux espaces publics urbains en tant qu'ils seraient menacés par le développement des enclaves résidentielles (Le Goix, 2001; Madoré, 2005) et des «espaces privés d'allure publique» (Dessouroux, 2003), comme les enclaves touristiques (Auvray, 2012), les centres commerciaux (Ghorra-Gobin, 2001) ou encore les parcs de loisirs (Didier, 2001).

9 La notion de publicisation (Tomas, 2001 ; Gasnier, 2006 ; Banos, Sabatier, 2010) est, quant à elle, plutôt mobilisée dans des travaux portant sur les espaces ruraux ou périurbains (espaces agricoles, espaces ouverts au sens large), en lien avec le développement des 
loisirs de plein air. Les espaces ouverts situés en périphérie des villes, bien que souvent de propriété privée, sont fréquentés de manière croissante par les habitants des villes, ce qui tend à les transformer à la fois en espaces publics et en objets de débat public (Clément, 2012). Certains travaux insistent sur la double dynamique de privatisation/publicisation, que ce soit entre types d'espaces - la publicisation des espaces ruraux répondrait à la privatisation des espaces urbains (Tomas, 2001) - ou au sein d'un même espace - la publicisation de l'espace rural entraînant des formes de privatisation, notamment une clôture plus systématique des propriétés (Banos, Sabatier, 2010).

\section{Dépasser la dichotomie public/privé}

10 La notion de privatisation et, dans une moindre mesure, celle de publicisation sont donc très utilisées, mais elles ne vont pas sans poser problème. Christian Dessouroux (2003) propose une analyse critique stimulante, fondée sur le constat que «l'usage abondant de la notion de privatisation se fait [...] à la faveur d'un grand flou sémantique ». Il invite à distinguer trois registres d'analyse souvent confondus par les travaux sur la privatisation (statut juridique des sols, modalités d'accès, régulation des usages) et cherche à « éclaircir les multiples dimensions du phénomène ». On retiendra en particulier la distinction qu'il opère entre privatisation juridique et restriction d'accès. On différenciera ainsi (i) la privatisation stricto sensu, processus juridique de mutation du droit de propriété et/ou des droits d'usage afférents (par le biais de conventions ou de concessions diverses), et (ii) les restrictions d'accès lato sensu, désignant la fermeture de certains espaces, indépendamment de leur statut juridique. De la même manière, si la notion de publicisation est intéressante, il faut adopter des précautions similaires à son égard et bien faire la part de ce qui tient au droit de propriété et de ce qui tient aux régulations concrètes de l'accès à l'espace.

11 En effet, les termes de privatisation et de publicisation sont intrinsèquement liés à la propriété et à la dichotomie public/privé. Or, la géographie du droit - courant qui s'intéresse tout particulièrement à l'expression spatiale de la norme juridique et qui a beaucoup travaillé le concept de propriété (Forest, 2009) - a mis en lumière le fait que la dichotomie public/privé est beaucoup moins stricte sur le terrain qu'en théorie. Nicolas K. Blomley (2005) montre, à partir de l'étude de pratiques de jardinage dans l'espace public, que des empiètements (encroachments) multiples interviennent entre espace public et espace privé, faisant de l'espace « an amalgam of the public and the private ». Les travaux de David Delaney (2009) invitent à dépasser l'idée d'une dichotomie public/ privédont la spatialisation serait évidente et insistent sur la complexité des manifestations spatiales de la propriété. La géographie du droit invite ainsi à prendre en compte la « résistance [...] de l'espace face aux ambitions normatives du droit » (Perret, 2009) et c'est précisément ce que la comparaison entre propriété foncière et accès concret entend faire.

\section{Présentation du terrain d'étude : les rives du lac d'Annecy}

Cette recherche s'appuie sur l'étude de cas du lac d'Annecy, dont le littoral très urbanisé fait l'objet d'une forte pression démographique, et dont la population présente un niveau socio-économique élevé, lui conférant une allure de riviera. Si ces caractéristiques sont 
communes à beaucoup de grands lacs alpins (Magni, Chinaglia, 2007), elles sont particulièrement marquées concernant le lac d'Annecy, au moins à l'échelle française ( tableau 1). On insistera ici sur les éléments de contexte qui éclairent les enjeux sociaux de partage de l'espace que l'on se propose d'étudier. Pour une présentation générale du lac, notamment sous ses aspects physiques, on se reportera aux travaux classiques qui l'ont bien décrit (Blanchard, 1916 ; Milon, 1939 ; Onde, 1944).

Tableau 1. Profil démographique et socio-économique du lac d'Annecy

\begin{tabular}{|c|c|c|c|c|c|}
\hline & \multirow[b]{2}{*}{$\begin{array}{l}\text { Lac } \\
\text { d'Annecy }\end{array}$} & \multicolumn{4}{|c|}{ Données comparatives } \\
\hline & & $\begin{array}{l}\text { Léman } \\
\text { (partie } \\
\text { française) }\end{array}$ & $\begin{array}{l}\text { Lac } \quad d u \\
\text { Bourget }\end{array}$ & $\begin{array}{l}\text { Savoie/ } \\
\text { Haute- } \\
\text { Savoie }\end{array}$ & $\begin{array}{l}\text { France } \\
\text { Métropolitaine }\end{array}$ \\
\hline \multicolumn{6}{|c|}{ Caractéristiques physiques du lac } \\
\hline Superficie (Km2) & 26,4 & 234 & 44 & - & - \\
\hline Périmètre (km) & 38 & 53 & 42,6 & - & - \\
\hline Profondeur moyenne (m) & 41,5 & 152,7 & 85 & - & - \\
\hline Altitude (m) & 447 & 372 & 232 & - & - \\
\hline \multicolumn{6}{|c|}{ Profil démographique des communes riveraines (avant les fusions de communes*) } \\
\hline $\begin{array}{l}\text { Nombre de communes } \\
\text { riveraines }\end{array}$ & 9 & 16 & 11 & - & - \\
\hline $\begin{array}{l}\text { Population totale des } \\
\text { communes } \\
(2012)\end{array}$ & 91299 & 73238 & 43889 & - & - \\
\hline $\begin{array}{l}\text { Évolution 2007-2012 de la } \\
\text { population des communes } \\
\text { riveraines }\end{array}$ & $1,3 \%$ & $9 \%$ & $5,5 \%$ & $5,9 \%$ & $2,6 \%$ \\
\hline $\begin{array}{l}\text { Densité de population } \\
\text { moyenne des } \\
\text { riveraines en } \mathrm{hbts} / \mathrm{km} 2 \\
(2012)\end{array}$ & 627 & 513 & 294 & 121,2 & 116,5 \\
\hline $\begin{array}{l}\text { Part du linéaire littoral } \\
\text { artificialisé } \\
\text { Corinne Land Cover, 2006) }\end{array}$ & $70,6 \%$ & $65,8 \%$ & $37,5 \%$ & - & - \\
\hline \multicolumn{6}{|c|}{ Profil socio-économique des communes riveraines } \\
\hline $\begin{array}{l}\text { Part des résidences } \\
\text { secondaires dans le total } \\
\text { des logements }(2012)\end{array}$ & $19,9 \%$ & $20,7 \%$ & $15,7 \%$ & $30,9 \%$ & $9,5 \%$ \\
\hline
\end{tabular}




\begin{tabular}{|l|l|l|l|l|l|}
\hline $\begin{array}{l}\text { Part de diplômés du } \\
\text { supérieur dans la } \\
\text { population > } 15 \text { ans non } \\
\text { scolarisée (2012) }\end{array}$ & $41,5 \%$ & $31,7 \%$ & $32,9 \%$ & $27,9 \%$ & $26,4 \%$ \\
\hline $\begin{array}{l}\text { Part de foyers fiscaux } \\
\text { imposables (2012) }\end{array}$ & $62,7 \%$ & $54,9 \%$ & $55,8 \%$ & $52,8 \%$ & $47,3 \%$ \\
\hline
\end{tabular}

*Plusieurs communes riveraines du lac d'Annecy ont fait l'objet d'une fusion. Au $7^{\mathrm{er}}$ janvier 2016, Talloires, commune située sur la rive est du lac, a fusionné avec Montmin, commune auparavant non riveraine. Au $7^{\text {er }}$ janvier 2017, Annecy et Annecy-le-Vieux, toutes deux riveraines du lac, ont fusionné avec 4 autres communes, auparavant non riveraines.

Sources : Alpines Lakes Network (2007) et Montuelle et Clémens (2015) pour les caractéristiques physiques; INSEE, 2012 pour les données statistiques ; Union européenne - SoeS, CORINE Land Cover, 2006 pour l'artificialisation

Conception et réalisation : A. Nikolli, 2016

\section{Un littoral peuplé et urbanisé}

Ce lac se situe en Haute-Savoie (figure 1), département marqué par une urbanisation forte, rapide et continue depuis le milieu du $\mathrm{XX}^{\mathrm{e}}$ siècle. La population de l'aire urbaine d'Annecy a augmenté de $12 \%$ entre 1999 et 2007, pour atteindre 220000 habitants en 2012 (INSEE, 2012). Au-delà de la seule aire urbaine d'Annecy, c'est tout le bassin versant du lac qui connaît une forte croissance démographique, de $13 \%$ entre 1990 et 2011 (Montuelle et Clemens, 2015). La population des communes riveraines du lac s'élève en 2012 à plus de 90000 habitants, pour une densité de population moyenne de 627 hab/ $\mathrm{km}^{2}$ (tableau 1). Ce dynamisme démographique entraîne une forte pression urbaine et foncière sur les rives du lac, qui connaissent une flambée des prix immobiliers (Laslaz et al., 2015). Le littoral lacustre est largement artificialisé (tableau 1) et le paysage offert par le lac est marqué par une urbanisation diffuse très prégnante (DIREN Rhône-Alpes, 2006a, 2006b). 
Figure 1. Localisation et présentation du terrain d'étude

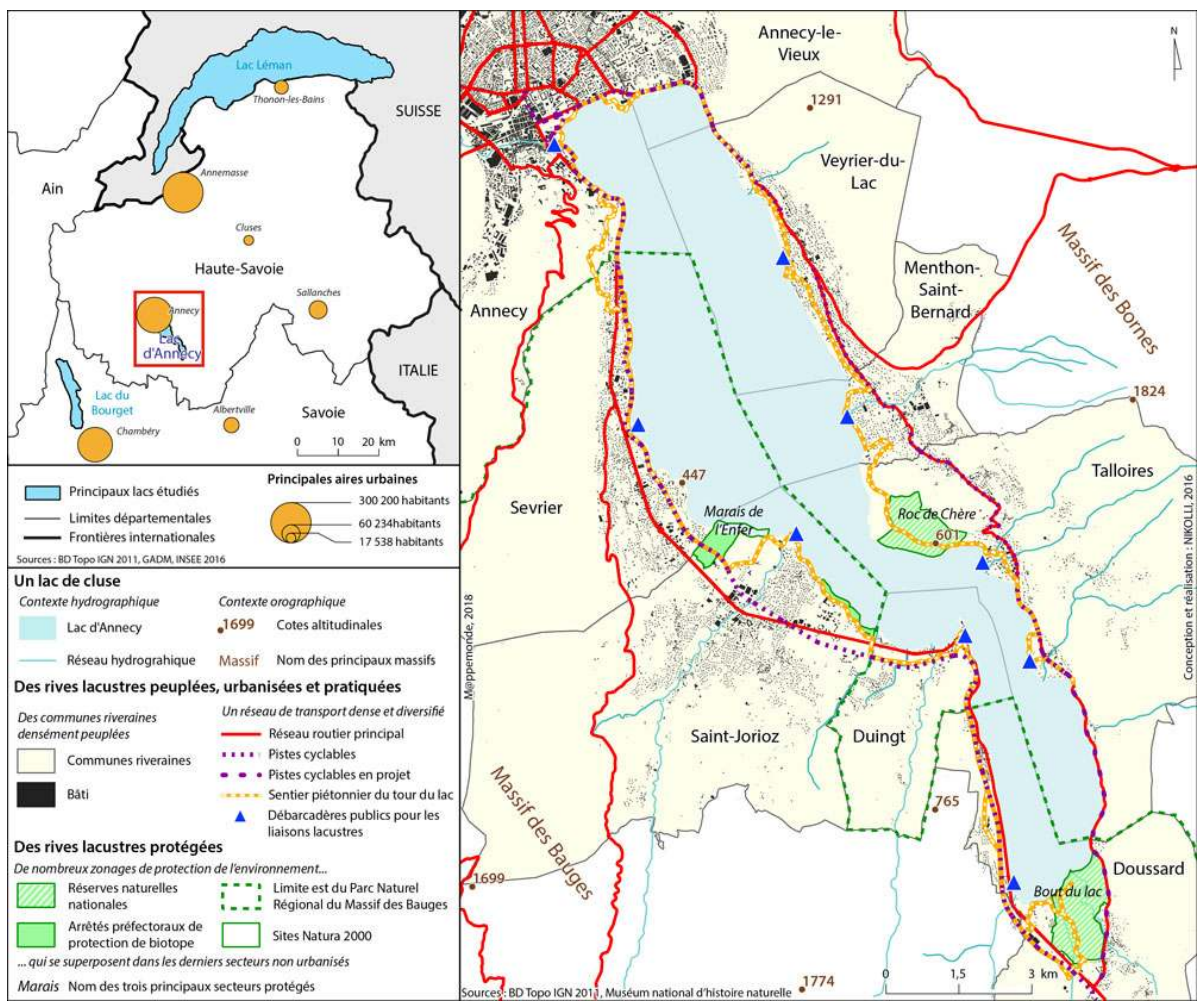

Conception et réalisation : A. Nikolli, 2016

\section{Une riviera lacustre}

14 Ce lac se caractérise par le niveau socio-économique élevé de la population riveraine, si on le compare aux autres grands lacs alpins français, à la moyenne des deux départements savoyards ou à la moyenne nationale (tableau 1). La rive est, en particulier, présente un paysage de riviera, au sens de «côte touristique dominée par la résidence riche » (Brunet et al., 1993). Elle est en effet marquée par des grandes propriétés, des villas luxueuses et des établissements gastronomiques réputés, notamment à Talloires et à Menthon-Saint-Bernard, en lien avec une tradition de villégiature élitaire datant du début du XXe siècle (Miège, 1933). Cette rive présente ainsi un profil socio-économique supérieur à la rive ouest avec une moyenne de $48 \%$ de diplômés de l'enseignement supérieur et $66 \%$ de foyers fiscaux imposables, contre respectivement $38 \%$ et $62 \%$ en rive ouest.

\section{Un terrain de jeu pour la population urbaine}

Le lac d'Annecy est très fréquenté, que ce soit par la population locale, par les excursionnistes ou par les touristes. Les loisirs de nature pratiqués dans, sur et autour du lac sont nombreux: promenade, vélo, baignade, navigation de plaisance, croisières lacustres, sports nautiques motorisés ou non, pêche amateur, événements (Montuelle, Clemens, 2015). La fréquentation globale est difficile à chiffrer, mais on dispose de données concernant certaines activités: le lac totaliserait 2,7 millions de visiteurs annuels (Office de tourisme du lac d'Annecy, 2016), la plaisance privée représente 
2000 bateaux, les compagnies de bateaux à passagers transportent en moyenne 155 000 personnes par saison, les pêcheurs amateurs sont près d'un millier (Montuelle, Clemens, 2015) tandis que la fréquentation cycliste autour du lac a été estimée à 1 300 vélos les jours de pointe (enquête menée en août 2004 par le Conseil général).

\section{Un joyau paysager sous haute protection}

Face à l'urbanisation des rives et à la forte fréquentation du lac, l'espace lacustre fait l'objet de toute une série de dispositifs de protection environnementale et paysagère (Natura 2000, réserves naturelles, politique départementale des espaces naturels sensibles [ENS], arrêtés préfectoraux de protection de biotope). La plupart des zonages de protection environnementale se superposent dans les trois mêmes secteurs, qui constituent les dernières coupures d'urbanisation autour du lac: le Roc de Chère, petit massif forestier qui tombe en falaise dans le lac, le marais de l'Enfer et le marais du Bout du lac (figure 1). Du point de vue foncier, le lac fait l'objet depuis 1976 d'une zone de préemption départementale dans le cadre des ENS, ainsi que de la politique d'acquisition du Conservatoire du littoral, qui a identifié 6 sites d'intervention (460 ha, dont 112 ha ont déjà été acquis) ${ }^{2}$. Enfin, l'urbanisation du rivage lacustre est strictement encadrée par deux textes principaux : la loi Montagne et la loi Littoral ${ }^{3}$, dont la superposition fait d'ailleurs débat (Joye, 2012). Ces deux lois, qui posent le principe de construction en continuité avec l'urbanisation existante et imposent une bande inconstructible $(300 \mathrm{~m}$ pour la loi Montagne, $100 \mathrm{~m}$ pour la loi Littoral), n'ont en revanche pas d'implication directe en matière d'accès aux rives. Si la loi Littoral réglemente sur l'accès aux rivages maritimes, en confirmant la servitude de passage des piétons le long du littoral (SPPL) mise en place en 1976, et en établissant une servitude transversale permettant de relier la voie publique au rivage, elle ne dit rien des servitudes de passage autour des lacs, lesquelles relèvent du droit du domaine public fluvial.

\section{Un lac « privatisé »?}

17 Dans ce contexte de grande fréquentation, qui a pu faire craindre une "saturation » à certains gestionnaires (Vernex, 1996a), le lac fait l'objet d'une forte demande d'accès, dont témoignent les densités humaines observées sur les rives en période estivale et les tensions sociales suscitées par les projets d'aménagement concernant le tour du lac (Nikolli, 2016). Or, cette demande d'accès se confronte à un espace «fini », en partie sous statut de protection, et largement contraint par la topographie, les infrastructures de transport et l'urbanisation résidentielle (figure 1). Cette dernière est souvent perçue comme une "privatisation» du lac (Estève, 1984; Vernex, 1989), notion largement invoquée dans le débat public local, souvent pour critiquer le fait que la présence de propriétés privées restreint l'accès du public au lac sur de longs linéaires. Pour autant, la notion n'est jamais définie précisément et elle est rarement mesurée avec rigueur. Aucun chiffre officiel n'est communiqué et la seule source scientifique disponible, qui tire d'une étude cadastrale un taux de privatisation de 46,2\%, date des années 1980 (Estève, 1984). De leur côté, les associations locales se livrent une bataille de chiffres : un taux de $41 \%$ de rives privées, mesuré de manière précise par une association environnementale ${ }^{4}$, s'oppose à un taux de $27 \%$, avancé par une association de propriétaires riverains, laquelle ne précise pas sa méthode de calcull ${ }^{5}$. De ce point de vue, les lacs alpins, et en particulier le lac d'Annecy, se différencient des espaces ruraux et périurbains pour lesquels les enjeux 
d'accès pour les pratiques récréatives ont été bien étudiés. Ici, ce ne sont pas les usages traditionnels tels que la chasse ou l'agriculture qui entrent en tension avec les pratiques récréatives en plein essor, mais bien l'urbanisation résidentielle sous forme de riviera, qui tend à restreindre l'accès du public aux rives. Les lacs alpins se distinguent en outre des rivages maritimes, dans la mesure où la nature juridique du droit d'accès aux rives lacustres est très différente de celle du droit d'accès au littoral maritime ${ }^{6}$.

\section{Cartographie de la propriété foncière et de l'accès aux rives : méthode}

\section{Propriété foncière et lacs : ce que dit le droit}

Pour faire le point sur l'accès aux rives des lacs alpins, le travail cartographique est incontournable, car le droit ne norme que très partiellement la situation. Le lac d'Annecy, comme le Léman et le Bourget, est un lac domanial qui relève du domaine public fluvial (DPF) (Davignon, 1979). Comme tout bien du domaine public, il est donc imprescriptible, inaliénable et librement accessible au public, en vertu du Code général de la propriété des personnes publiques (CGPPP) (Auby et al., 2016). Mais le régime de domanialité publique ne concerne que le plan d'eau, et non les rives. La limite entre le domaine public fluvial et les terrains riverains est définie par « la hauteur des eaux coulant à pleins bords avant de déborder » (art. L-2111-9 du CGPPP). Cette cote des plus hautes eaux ordinaires doit être établie par arrêté préfectoral ${ }^{7}$ et la limite du DPF est alors constituée par l'intersection entre la rive et le plan virtuel déterminé par la cote (figure 2). On notera que cette cote n'a jamais été fixée pour le lac d'Annecy, dont le DPF n'a donc pas de limites officielles8. Au-delà de cette limite, plus ou moins précise selon qu'elle a été légalement fixée ou non, les terrains riverains d'un lac domanial n'ont pas de statut de propriété prédéterminé : ils peuvent relever de la propriété privée comme de la propriété publique (domaine public ou domaine privé des personnes publiques ${ }^{9}$ ). Ils sont théoriquement grevés d'une servitude dite de marchepied, qui oblige les propriétaires à laisser libre le passage sur une largeur de 3,25 mètres à partir de la limite du DPF, quel que soit le statut de propriété des terrains, au bénéfice de tous les piétons (art. L-2131-2 du CGPPP) (figure 2). Néanmoins, il faut noter que cette servitude n'est pas systématiquement respectée par les propriétaires riverains des grands lacs haut savoyards. C'est tout particulièrement le cas autour du lac d'Annecy, où l'immense majorité des propriétés riveraines est close de haies, de murs ou de grillages qui se prolongent jusqu'à l'eau, tandis que la servitude est davantage respectée sur la rive française du Léman. 
Figure 2. Délimitation théorique du domaine public fluvial et de la servitude de marchepied

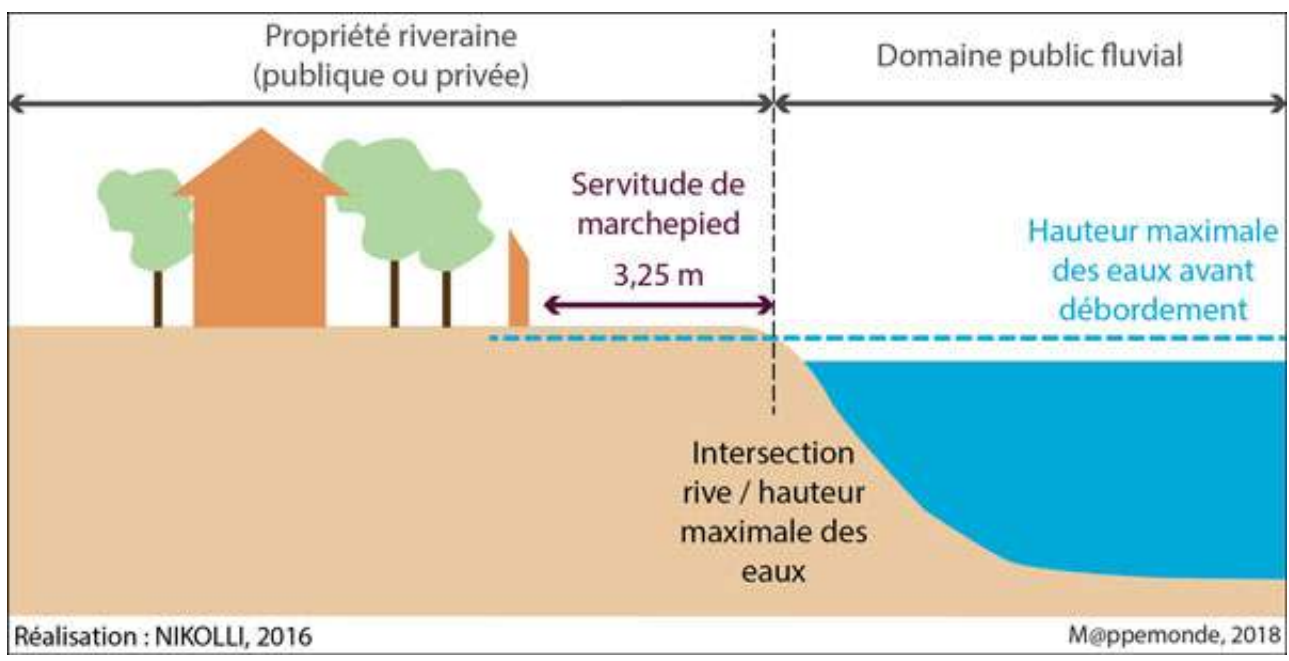

Conception et réalisation : A. Nikolli, 2016

\section{Cartographie de la propriété foncière} répartition entre espaces publics et privés autour du lac d'Annecy. La cartographie de la propriété foncière des terrains riverains $d u$ lac a été réalisée dans un système d'information géographique (SIG), sur la base des données cadastrales. D'une part, les parcelles cadastrées riveraines du lac ont été isolées et listées. Pour chacune de ces 825 parcelles, une enquête cadastrale a permis d'identifier le type de propriété (publique ou privée $)^{10}$. Les données de propriété ont ensuite été intégrées au SIG et ont permis de calculer les linéaires de rives relevant de parcelles privées et ceux relevant de parcelles publiques. D'autre part, les linéaires de rives relevant du domaine non cadastré ont été numérisés. Le domaine non cadastré doit être distingué du domaine public, dans la mesure où la non-cadastration ne signifie pas ipso facto que le terrain en question relève du domaine public, comme on le croit souvent (DGFIP, 2016). Par souci de rigueur, on a donc pris soin de distinguer le domaine non cadastré de la propriété publique cadastrée. Néanmoins, au regard des types d'espace non cadastrés relevés autour du lac d'Annecy (routes, quais, espaces verts urbains...), il est possible d'assimiler les linéaires non cadastrés à la propriété publique.

\section{Cartographie des modalités concrètes d'accès aux rives}

La cartographie de l'accès concret aux rives a également été effectuée dans un SIG, sur la base d'observations de terrain confrontées à des photographies aériennes. Ces observations de terrain ont consisté à tenter de longer au maximum le littoral lacustre et cherchent donc à documenter l'accès aux rives d'un point de vue linéaire. Cela a permis de repérer (i) à quels endroits on vient buter sur des espaces clos par divers dispositifs spatiaux de fermeture (haies, grillages...) et (ii) quels types d'espaces clos restreignent l'accès aux rives. Cette démarche de terrain cherchait à mesurer la possibilité concrète de longer la rive, dans la mesure où l'on se place ici au «niveau spatial de la rencontre physique entre l'homme et le rivage » (Hoffer, 2013). 
Pour cette cartographie, cinq catégories d'accès ont été retenues :

- L'accès libre désigne les espaces librement et gratuitement accessibles, sans condition ni restriction d'aucune sorte (quais publics, espaces verts urbains...).

- L'accès conditionné comprend les espaces accessibles au public sous réserve du paiement d'un droit d'entrée (plages municipales), de la consommation d'un service (restaurants pieds dans l'eau, complexes de loisirs...) ou de toute autre condition.

- L'accès privatif regroupe les secteurs où la rive n'est pas accessible au public du fait de dispositifs spatiaux de fermeture relevant du marquage de la propriété privée (clôtures, haies...).

- L'accès physiquement difficile cible les linéaires où la rive est inaccessible du fait de la topographie (falaises) ou de formations végétales (roselières).

- L'accès interdit correspond aux dispositifs institutionnels interdisant explicitement l'accès, en l'occurrence pour des raisons de protection de l'environnement.

\section{Déconnexion propriété/accès et enjeux sociaux de partage de l'espace sur un littoral lacustre convoité}

\section{Une propriété publique majoritaire, mais d'importantes restrictions d'accès}

La cartographie de la propriété foncière (figure 3) permet d'établir que 23,8 \% du linéaire littoral du lac d'Annecy sont constitués de parcelles publiques, auxquels on peut ajouter $37 \%$ de linéaire non cadastré assimilé à la propriété publique, soit un total de $60,8 \%$ du littoral sous maîtrise foncière publique. Ce sont donc $39,2 \%$ du linéaire qui se trouvent aux mains de personnes privées. On remarque une forte variabilité d'une commune à l'autre, entre des communes où la quasi-intégralité, voire l'intégralité, du linéaire relève de la propriété publique (Annecy-le-Vieux, Annecy, Saint-Jorioz) et d'autres où $80 \% \mathrm{du}$ linéaire appartiennent à des personnes privées (Veyrier-du-Lac).

La cartographie de l'accès concret aux rives (figure 4) met en lumière un taux de 48,5 \% de rives en libre accès, contre $51,5 \%$ du linéaire qui font l'objet de restrictions d'accès. Au sein de ces restrictions d'accès, ce sont les restrictions privatives qui sont les plus prégnantes, avec $34 \%$ du linéaire total. L'accès physiquement difficile représente $8,4 \% \mathrm{du}$ linéaire, l'accès conditionné $6,9 \%$ et l'accès interdit $2,2 \%$. De la même manière que pour la propriété foncière, on observe une forte variabilité intercommunale, entre des communes où l'intégralité du linéaire est librement accessible et des communes où le linéaire inaccessible l'emporte largement, qu'il soit lié au poids des restrictions privatives (Veyrier-du-Lac) ou des restrictions physiques (Talloires). 
Figure 3. Propriété privée et propriété publique sur les rives du lac d'Annecy

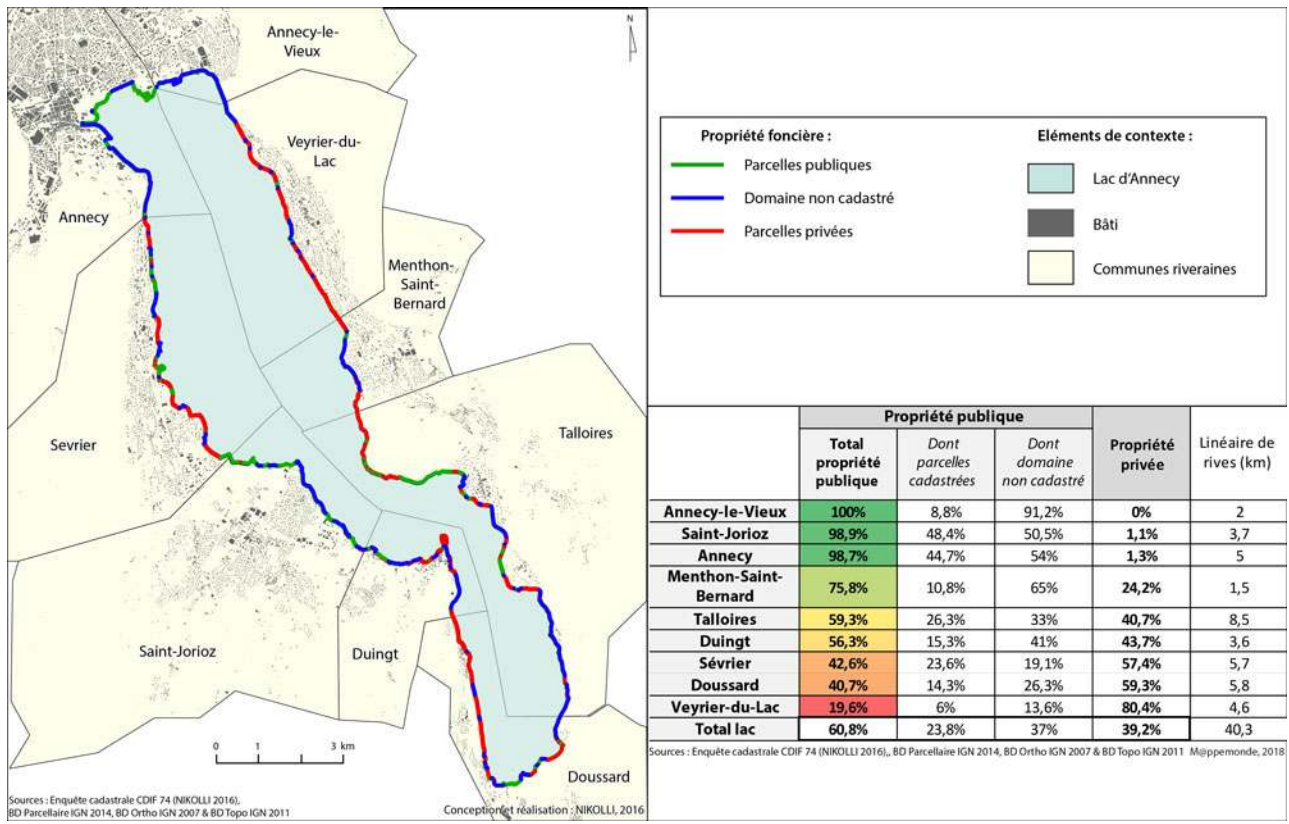

Conception et réalisation : A. Nikolli, 2016

Figure 4. Accès concret aux rives du lac d'Annecy

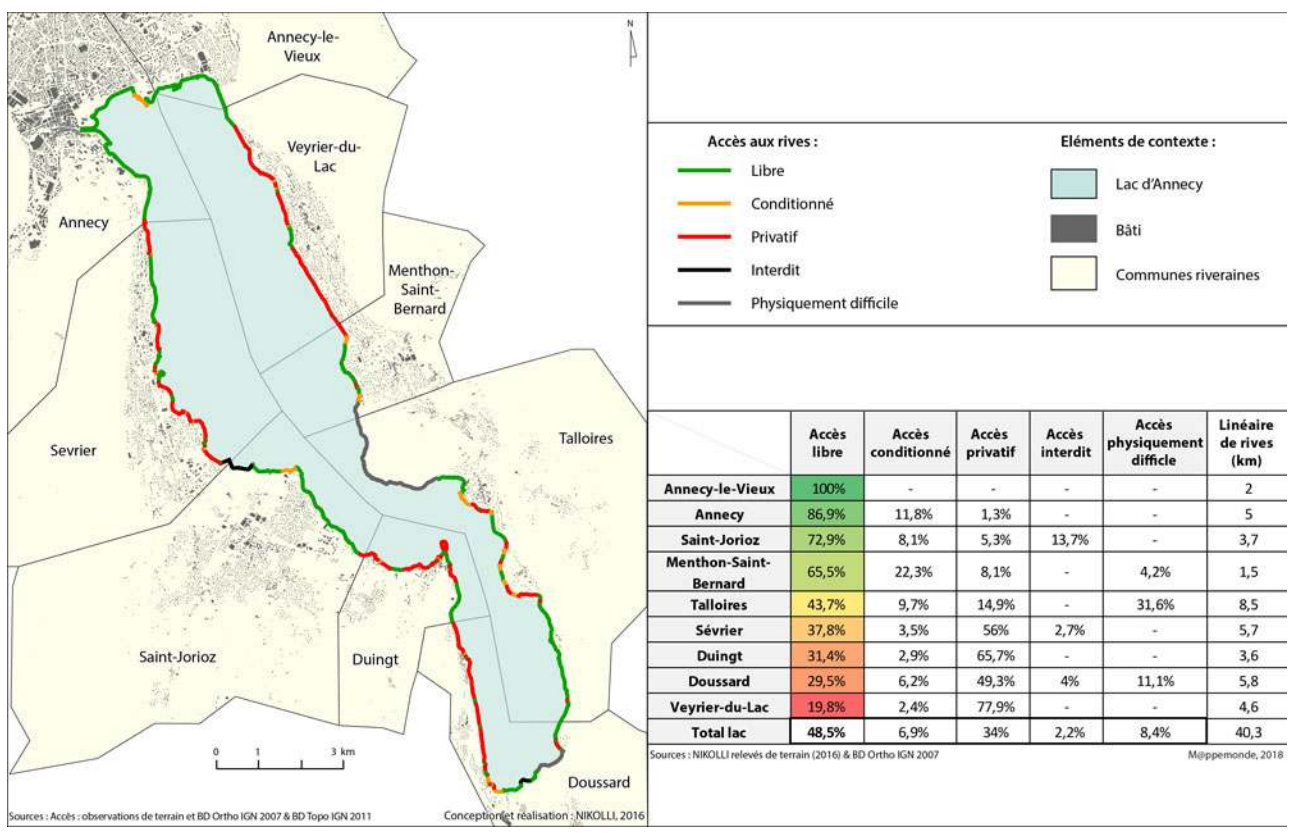

Conception et réalisation : A. Nikolli, 2016

\section{Des empiètements multiples et réciproques entre public et privé}

Avant de croiser les résultats de ces deux cartographies, quelques précisions de vocabulaire s'imposent. Les représentations spatiales dominantes considèrent la dichotomie public/privé comme simple et binaire (Delaney, 2009). De ce point de vue, l'espace de propriété publique est supposé être accessible à tous et l'espace de propriété 
privée est supposé être réservé à un usage privatif, excluant dès lors le public. Pour désigner les situations où le sens commun n'est pas démenti, on parlera d'articulation « cohérente » entre propriété et accès, cohérence appréciée au regard des archétypes de l'espace public (rues, places: propriété publique et accès libre) et des archétypes de l'espace privé (maison: propriété privée et accès privatif). À l'inverse, on parlera de déconnexion pour désigner les situations d'empiètement entre propriété et accès. Le terme d'empiètement (encroachment) est emprunté à l'article de Nicolas K. Blomley déjà cité (Blomley, 2005). En français, le terme désigne le « fait de s'étendre, de mordre, de déborder sur " (Trésor de la langue française). Il permet donc de désigner les situations où telle modalité d'accès, caractéristique d'une des deux modalités de propriété, s'étend sur un espace relevant de l'autre modalité de propriété (exemple: un espace de propriété privée qui serait librement accessible, car l'accès libre renvoie à l'archétype de l'espace public, et non de l'espace privé).

On observe donc une certaine déconnexion entre les deux variables, dans la mesure où, si $60,8 \%$ du linéaire littoral relèvent de la propriété publique, seuls $48,5 \%$ sont librement accessibles à tous. Le croisement des deux séries de données montre ainsi que seuls 76,5\% du linéaire total correspondent à des combinaisons dans lesquelles la propriété foncière et la régulation des accès sont en cohérence : $46,4 \%$ de linéaires de propriété publique en accès libre et 30,1\% de linéaires de propriété privée en accès privatif. Restent $23,5 \% \mathrm{du}$ linéaire total, où l'on retrouve tous les croisements possibles entre les deux catégories de propriétés et les cinq catégories d'accès, qui mettent en lumière l'existence d'empiètements entre propriétés et accès (figure 5).

Figure 5. Empiètements entre espaces publics et espaces privés

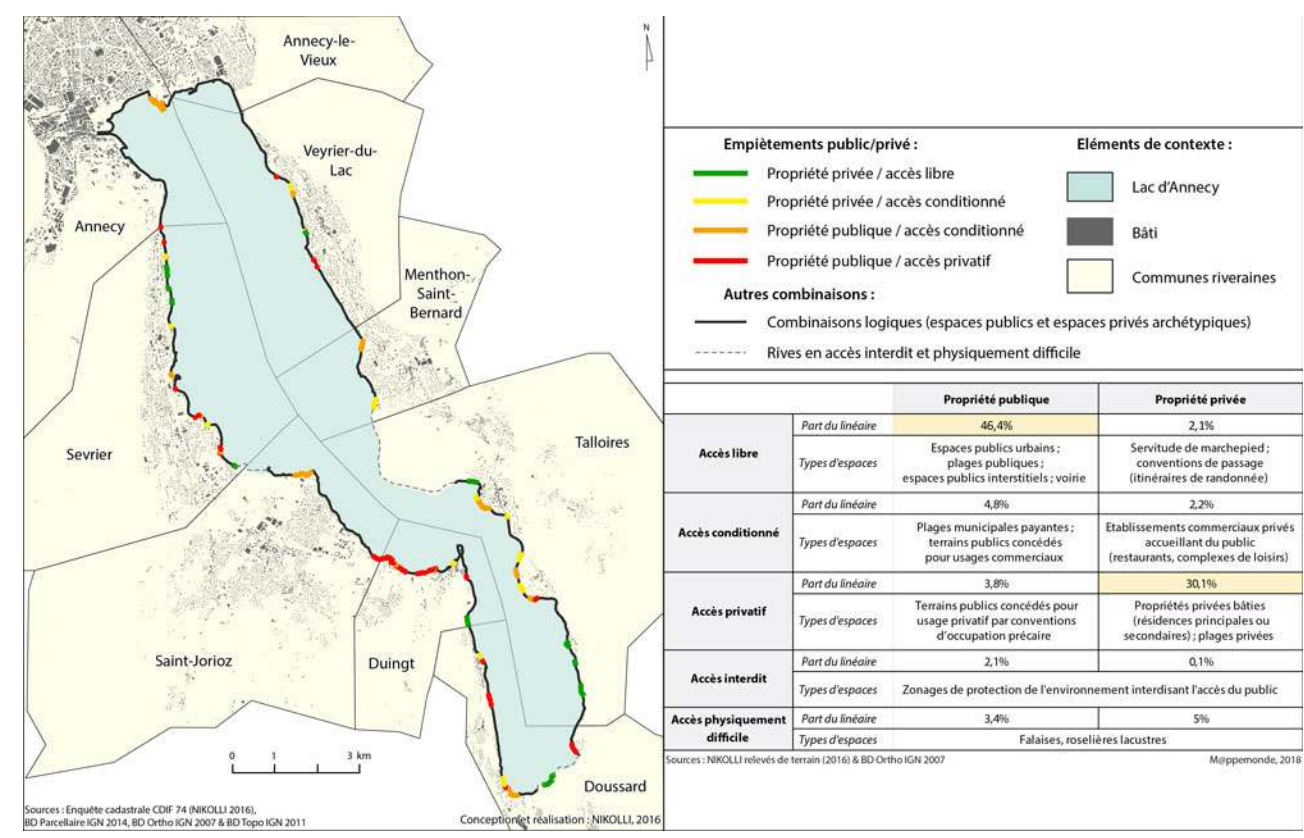

Conception et réalisation : A. Nikolli, 2016

On peut ainsi distinguer des cas où accès et propriété sont en cohérence et d'autres où les deux données tendent à être déconnectées l'une de l'autre (figure 6). Les espaces où la propriété et l'accès sont en cohérence (espaces publics et espaces privés archétypiques) sont certes majoritaires. Pour autant, l'axe de déconnexion montre que les espaces de 
propriété publique ne sont pas systématiquement accessibles à tous (privatisation de l'accès aux espaces de propriété publique) et que les espaces de propriété privée ne sont pas nécessairement inaccessibles au public (publicisation de l'accès aux espaces de propriété privée). Les catégories " accès interdit » et « accès physiquement difficile », qui rendent le critère de la propriété relativement négligeable, sont volontairement laissées de côté, pour se concentrer sur les croisements entre propriété publique, propriété privée et leurs attributs conventionnels respectifs. Précisons en outre que l'on s'autorise à mobiliser les notions de privatisation et de publicisation, critiquées plus haut dans leur acception extensive, dans la mesure où l'on se concentre ici sur le droit de propriété et sur les droits d'usage qui lui sont afférents, donc sur leur acception juridique.

Figure 6. Une répartition entre espace public et espace privé à géométrie variable

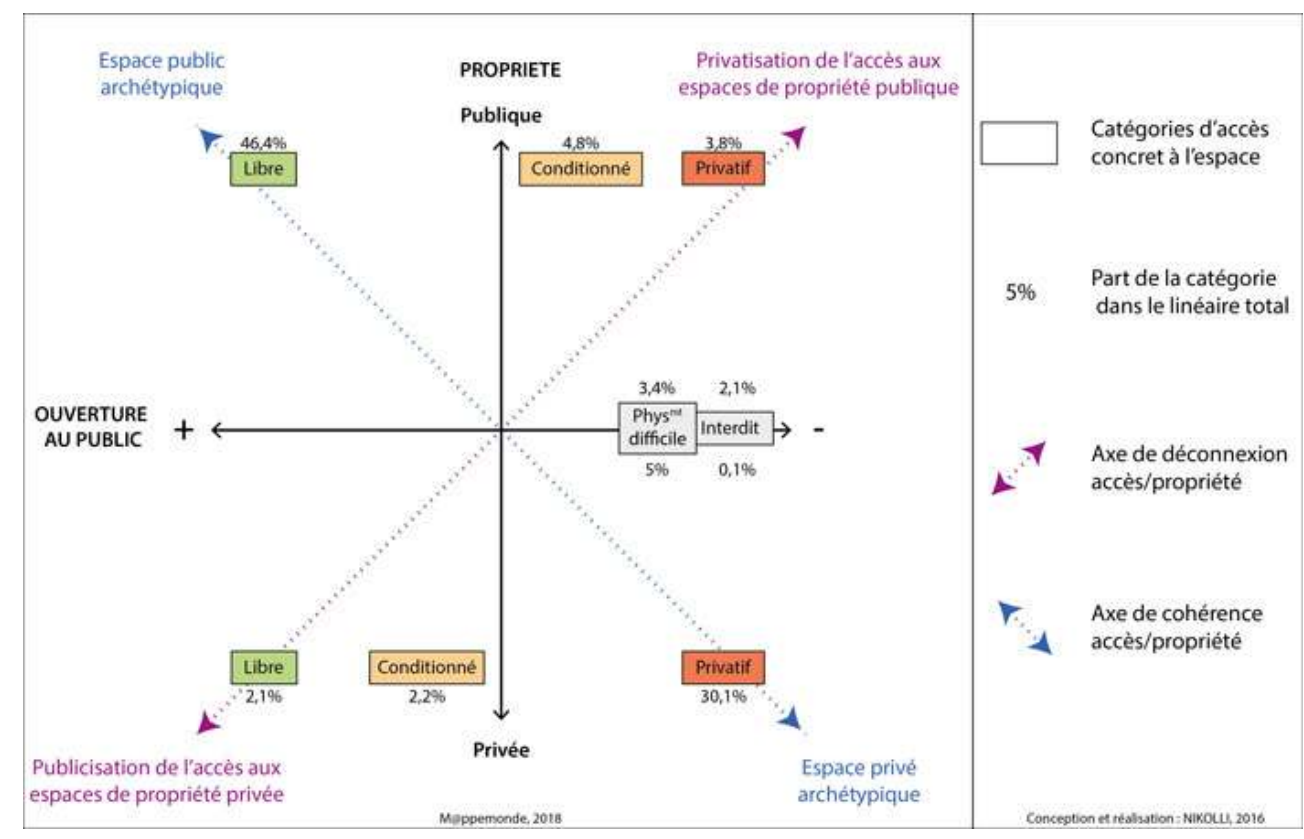

Conception et réalisation : A. Nikolli, 2016

\section{Entre privatisation et publicisation : enjeux de partage de l'espace sur un littoral convoité}

L'axe de déconnexion met donc en lumière une double dynamique de privatisation de l'accès aux espaces de propriété publique et de publicisation de l'accès aux espaces de propriété privée, qui est certes minoritaire en termes de linéaire concerné, mais qui concentre les enjeux sociaux de partage de l'espace sur un littoral lacustre convoité.

Les phénomènes de privatisation sur les rives $\mathrm{du}$ lac constituent un sujet particulièrement sensible. Dans le débat public local, le terme est utilisé pour critiquer le fait que les propriétés privées pieds dans l'eau restreignent l'accès aux rives. L'existence de propriétés jouissant du privilège spatial d'un accès privatif au lac est vécue comme une prédation de l'espace public. Cette acception du terme de « privatisation » est néanmoins abusive, dans la mesure où rien ne permet d'affirmer a priori que ces propriétés ont privatisé un espace relevant auparavant de la propriété publique. En revanche, le travail cartographique atteste d'un cas bien spécifique de privatisation d'un espace de propriété 
publique : à Duingt, des terrains du domaine privé de l'État (huit parcelles représentant $15 \%$ du linéaire littoral de la commune) sont concédés depuis la première moitié du $\mathrm{XX}^{\mathrm{e}}$ siècle à des particuliers qui y ont construit des maisons. Il s'agit là d'un cas de privatisation juridique, au sens où le droit d'usage d'un terrain public a été concédé à des personnes privées (par le biais de conventions d'occupation dite précaire, mais régulièrement renouvelées depuis près d'un siècle) et a, dès lors, entraîné une restriction d'accès durable pour le public. Celle-ci est d'autant plus marquée que les propriétaires de ces terrains ne respectent pas la servitude de marchepied, qui autorise théoriquement tous les piétons à circuler sur une bande de $3,25 \mathrm{~m}$ à partir de la limite du DPF. Il est d'ailleurs intéressant de constater que ce cas effectif de privatisation est très peu connu dans la société locale, et donc très peu contesté, alors que, à l'inverse, l'existence de plages municipales payantes ${ }^{11}$ (qui correspondent non pas à une privatisation, mais à un conditionnement de l'accès) cristallise les critiques. Un cas similaire existait jusqu'à une date récente dans la commune de Sevrier (des terrains publics concédés à des privés, mais bâtis de manière plus légère, sous forme de cabanes). L'État a récemment mis fin aux conventions d'occupation de manière à récupérer les terrains, qui ont été confiés au Conservatoire du littoral pour mener un projet de renaturation et de réouverture au public.

Une certaine dynamique de publicisation semble ainsi à l'œuvre autour du lac d'Annecy. Sa mise en lumière contraste avec la littérature en sciences sociales qui insiste sur une privatisation croissante de l'espace public (Decroly et al., 2003). Outre le cas de Sevrier (qui ne constitue pas tant une publicisation de l'espace privé qu'une reconnexion entre propriété et accès), certains espaces de propriété privée se trouvent ouverts au public, par le biais de conventions de passage passées par les collectivités publiques avec les propriétaires (dans le cadre de la mise en place d'itinéraires de randonnée) ou en vertu de la servitude de marchepied. Autour du lac d'Annecy, l'application concrète de cette servitude de passage réglementaire est rare (un linéaire de quelques centaines de mètres dans une seule des neuf communes riveraines). Sur la rive française du Léman, en revanche, son application est beaucoup plus commune : la servitude y est respectée sur $86 \%$ du littoral, bien que ce soit en pointillés ${ }^{12}$, à l'exception notable de la commune d'Anthy-sur-Léman où la servitude est appliquée de manière concertée par la municipalité sur l'intégralité du linéaire communal. La publicisation prend ici le double sens d'ouverture d'espaces de propriété privée au public et de question discutée dans le débat public, dans la mesure où l'application de cette servitude devient un enjeu social important depuis quelques années. À l'image du conflit qui agite les rives de l'Erdre, en Loire-Atlantique, depuis les années 1960 (Rollo et al., 2012), plusieurs associations locales commencent à militer pour l'application de cette servitude sur les rives des grands lacs alpins. Sur la rive française du Léman, le militantisme associatif est important: organisation de marches pour sensibiliser la population et mettre en pratique le droit de passage ouvert par la loi, cartographie participative de l'état de la servitude, sollicitation des élus... Sur le lac d'Annecy, le dynamisme social est moindre, mais les acteurs associatifs se saisissent néanmoins de la question, avec un mode opératoire essentiellement contentieux. 


\section{Conclusion}

La cartographie comparée de la propriété foncière et de l'accès concret à l'espace sur les rives du lac d'Annecy met ainsi en lumière une relative déconnexion entre les deux variables et l'existence d'empiètements, nombreux et réciproques, entre public et privé. La répartition entre espace public et espace privé apparait donc non pas comme une dichotomie stricte et exclusive, mais comme le résultat d'un agencement à géométrie variable entre des droits de propriété, des droits d'usage afférents et des pratiques spatiales. Si l'accès à l'espace public peut faire l'objet de dynamiques de privatisation, comme la littérature en sciences sociales l'a bien montré, le cas du lac d'Annecy suggère que celles-ci ne sont pas nécessairement irréversibles (un espace public qui a été privatisé peut se trouver republicisé) et qu'elles ne contredisent pas un processus concomitant de publicisation de l'accès à des espaces de propriété privée. Sous l'effet de mobilisations sociales émergentes, les enjeux d'accès au lac alimentent une publicisation de l'espace lacustre, dans une acception tant pragmatique que sociopolitique du terme. Cette recherche montre ainsi comment le droit (en l'occurrence, de propriété) se traduit en actes dans l'espace, et se trouve au passage réinterprété, en fonction des pratiques spatiales et des aspirations sociales. Mais, réciproquement, l'espace est influencé par le droit et le contexte législatif en cours d'évolution tend à changer la donne autour des grands lacs alpins. La servitude de marchepied a été renforcée en 2015, et la loi permet désormais aux collectivités locales ainsi qu'aux associations d'usagers de saisir l'autorité gestionnaire de manière à en faire délimiter l'emprise ${ }^{13}$. Les conditions du partage de l'espace sur les rives lacustres pourraient donc se trouver sensiblement modifiées à moyen terme, dans des proportions et selon des modalités variables d'un lac à un autre, en fonction des contextes sociaux, politiques et administratifs locaux.

\section{BIBLIOGRAPHIE}

AMALRIC M. (2005). Les zones humides, appropriations et représentations. L'exemple du Nord-Pas-deCalais. Thèse de doctorat, Lille, Université Lille I.

ARZUL G. (2008). Le renouveau du droit du domaine public fluvial. Paris : Éd. Johanet, 503 p.

ISBN 978-2-9000-8681-0

AUBY J.-M., BON P., AUBY J.-B., TERNEYRE P. (2016). Droit administratif des biens : domaine public et privé, travaux et ouvrages publics, expropriation. $7^{\mathrm{e}}$ éd., Paris : Dalloz, $720 \mathrm{p}$.

AUVRAY B. (2012). «L'enclave touristique : déclinaison exotique de la communauté fermée?» URBIA, $\mathrm{n}^{\circ} 14$.

BANOS V., SABATIER B. (2010). « Les espaces périurbains non bâtis en France : entre publicisation "urbaine" et privatisation "rurale" ? " Articulo. Journal of Urban Research, n 3. 
BARTHEL P.-A. (2006). « Urbanités complexes : la fabrique des lieux “publics” aux Berges du lac de Tunis ». Espaces et sociétés, vol. 127, n 4, p. 129-144.

BERDOULAY V., DA COSTA GOMES P.-C., LOLIVE J. (2004). L'espace public à l'épreuve : régressions et émergences. Pessac: Maison des sciences de l'homme d'Aquitaine, $224 \mathrm{p}$.

BLANCHARD R. (1916). «Annecy. Esquisse de géographie urbaine ». Recueil des travaux de l'institut de géographie alpine, t. 4, $\mathrm{n}^{\circ}$ 4, p. 369-463.

BLOMLEY N.K. (2005). "Flowers in the bathtub: boundary crossings at the public-private divide". Geoforum, vol. 36, n 3, p. 281-296.

BOURDEAU P., MAO P., CORNELOUP J. (2011). « Les sports de nature comme médiateurs du "pas de deux" ville-montagne. Une habitabilité en devenir ? » Annales de géographie, vol. 680, $\mathrm{n}^{\circ} 4$, p. $449-460$.

BRUNET R., FERRAS R., THÉRY H. (1993). Les mots de la géographie. Montpellier/Paris : GIP Reclus/ Documentation Française, $518 \mathrm{p}$.

вUнот C. (2005). « Analyse comparative de la propriété foncière ». Norois, n 196, p. 81-90.

BUHOT C., GÉRARD Y., BRULAY F., CHOBLET C. (2009). Tensions foncières sur le littoral. Rennes : Presses universitaires de Rennes, 172 p. ISBN 978-2-7535-0859-0

CADORET A. (2011). «Quelle durabilité des formes de régulation des conflits littoraux?». VertigO, hors série, $\mathrm{n}^{\circ} 9$.

CLARIMONT S., LEICHNIG K. (2014). « La perception des espaces publics de nature. Paroles d'élus et d'usagers du Parc naturel urbain palois ». Revue géographique de l'Est, vol. 54, n 3-4.

CLEMENT C. (2012). La publicisation des espaces agricoles périurbains : du processus global à la fabrique du lieu. Thèse de doctorat, Montpellier 3,536 p.

сомву E. (2013). « Les discours de presse sur les reconquêtes du Rhône lyonnais (Le Progrès, 2003-2010)». Géocarrefour, vol. 88, n 1, p. 31-43.

COUIX G., LE ROY R. (1994). « Un modèle graphique du droit du littoral ». M@ppemonde, n² 2, p. 8. En ligne : https://www.mgm.fr/PUB/Mappemonde/M294/LITTORAL.pdf

DAVIGNON J.-F. (1979). « La condition juridique des lacs ». AJDA.

DAVIS M. (1992). "Fortress Los Angeles: the militarization of urban space”. In M. sORKIN (éd.), Variations on a theme park: The new American city and the end of public space, New York : Hill \& Wang, p. $154-180$.

DECROLY J.-M., DESSOUROUX C., VAN CRIEKINGEN M. (2003). « Les dynamiques contemporaines de privatisation des espaces urbains dans les villes européennes ». BELGEO, n 1, p. 3-20.

DELANEY D. (2009). « Le juridique, le spatial et la pragmatique de la construction de la réalité ». In P. FOREST (dir.), Géographie du droit. Épistémologie, développement et perspectives, Québec : Presses de l’Université de Laval, p. 117-135.

DELBAERE D. (2010). La fabrique de l'espace public. Ville, paysage et démocratie. Paris : Ellipses, 186 p. ISBN 9782729861582

DESSOURoux C. (2003). «La diversité des processus de privatisation de l'espace public dans les villes européennes ». BELGEO, n 1, p. 21-46.

DGFIP (2016). « Définitions et gestion du domaine non cadastré par la DGFiP ». Direction générale des Finances publiques/Conseil National de l'Information géographique. 
DIDIER S. (2001). « Parcs de loisirs et nouveaux espaces publics : le "Disneyland Resort" d'Anaheim (Californie) ». In C. GHORRA-GOBIN (dir.), Réinventer le sens de la ville. Les espaces publics à l'heure globale, Paris : L'Harmattan, p. 149-157.

DIREN RHONE-ALPES (2006a). « Grand lac d'Annecy. Unité paysagère 115 - HS ». Observatoire des paysages en Rhône-Alpes.

DIREN RHONE-ALPES (2006b). « Petit lac d'Annecy. Unité paysagère 119 - HS ». Observatoire des paysages en Rhône-Alpes.

ESTEVE R. (1984). Un test pour l'environnement : le lac d'Annecy. Mémoire de DEA, Lyon, Université Lyon 3, faculté de droit, $110 \mathrm{p}$.

FLEURY A. (2005). « Les rivages d'Istanbul : des espaces publics au cœur de la mégapole ». Géographie et cultures, vol. 52, p. 55-72.

FOREST P. (2009). «Géographie du droit : l'épissure de la norme et de l'espace ». In P. FOREST (dir.), Géographie du droit : épistémologie, développement et perspectives, Québec : Presses universitaires de Laval, p. 23-43.

GASNIER A. (2006). « De nouveaux espaces publics urbains ? Entre privatisation des lieux publics et publicisation des lieux privés ». Urbanisme, n³46, p. 70-73.

GERARDOT C. (2007). Fleuves et action urbaine : de l'objet à l'argument géographique. Le Rhône et la Saône $\grave{a}$ Lyon, retour sur près de trente ans de "reconquête » des fronts d'eau urbains. Thèse de doctorat, Lyon, Université Lyon 2, $513 \mathrm{p}$.

GHORRA-GOBIN C. (dir.) (2001). Réinventer le sens de la ville. Les espaces publics à l'heure globale. Paris : L'Harmattan, 265 p.

GRAVARI-BARBAS M. (1991). La mer retrouvée : Baltimore et autres reconquêtes de fronts d'eau urbains. Thèse de doctorat, Paris, Université Paris 4.

HOFFER O. (2013). Quand le littoral se ferme. Quelle gouvernance de l'accès et des usages de l'interface littorale dans les agglomérations d'Auckland, de Nouméa et de Port-Vila? Thèse de doctorat, Université de la Réunion, $410 \mathrm{p}$.

HRAFNKELSDOTTIR H. (2010). Public access to lakeshores in Iceland. Second cycle, A2E, Alnarp, Swedish University of Agricultural Sciences.

JOYE J.-F. (2012). « Loi Montagne, loi Littoral : situation des communes sous double contrainte ». L'écriture des PLU de montagne, fiche 6, GRIDAUH.

LARRIEU E. (1910). Le régime légal du lac d'Annecy. Thèse de doctorat, Paris, Université de Paris, 71 p. LASLAZ L., GAUCHON C., PASQUET O. (2015). Atlas Savoie Mont-Blanc : au carrefour des Alpes, des territoires attractifs. Paris : Autrement, $128 \mathrm{p}$.

LE CARO Y., KERGREIS S. (2007). « L'espace agricole comme espace public : accès récréatif et entretien du bocage en Bretagne ». In LE CARO Y., MADELINE P. ET PIERRE G. (dir.), Agriculteurs et territoires. Entre productivismes et exigences territoriales, Presses universitaires de Rennes, coll. « Espace et territoire », p. 231-250.

LE GoIX R. (2001). «Les “communautés fermées” dans les villes des États-Unis ». L’Espace géographique, vol. 30, n 1, p. 81-93.

LuSSAult M. (2003). « Espace public ». In J. LEVY ET M. LusSAUlT (dir.), Dictionnaire de la géographie et de l'espace des sociétés, p. 333-340. 
MADORE F. (2005). « La ségrégation sociale dans les villes françaises : réflexion épistémologique et méthodologique ». Cahiers de géographie du Québec, vol. 49, n 136, p. 45-60.

MAGNI D., CHINAGLIA N. (2007). "Alpine Lakes. A common approach to the characterization of lakes and their catchment area". Alpine Lakes Network.

MELIN H. (2015). « La pratique des sentiers périurbains de Balagne (Corse) par les habitants : marcher pour réinventer sa vi(ll)e ». Environnement Urbain/Urban Environment, vol. 9.

MERMET L., MOQUAY P. (dir.) (2002). Accès du public aux espaces naturels : outils d'analyse et méthodes de gestion. Paris : Hermès-Lavoisier, $393 \mathrm{p}$.

MEUR-FEREC C. (2007). «Entre surfréquentation et sanctuarisation des espaces littoraux de nature ». L'Espace géographique, t. 36, n 1, p. 41-50.

Michel C. (2003). L'accès du public aux espaces naturels, agricoles et forestiers et l'exercice du droit de propriété: des équilibres à gérer. Thèse de doctorat, ENGREF (AgroParisTech).

MIEGE J. (1933). « La vie touristique en Savoie ». Revue de géographie alpine, vol. 21-22, p. 749-817 (vol. 21) et p. 5-213 (vol. 22).

MILON F. (1939). « Le lac d'Annecy, étude géographique ». Annales de Géographie, vol. 48, n 272, p. $120-137$.

MITCHELL D. (2003). The right to the city. Social justice and the fight for public space. New York: Guilford Press, 270 p. ISBN 9781572308473

MONTUELLE B., CLEMENS A. (dir.) (2015). Le tour des grands lacs alpins naturels en 80 questions. Zone Atelier Bassin du Rhône et Observatoire des Lacs Alpins, Villeurbanne : GRAIE, 205 p.

MORI N., PAGON P., BRANCELJ A. (2007). Public lakeshore access and lakeside occupation. Ljubljana : National Institute of Biology.

NICOLAS L. (2008). «La plage de Beauduc : entre communitas et utopie ». Géographie et cultures, $\mathrm{n}$ 67, p. 93-112.

NIKOLLI A. (2016). Les grands lacs alpins, des espaces publics? Le «droit au lac " face aux restrictions d'accès aux rives lacustres. Étude de cas du lac d'Annecy, de la rive française du Léman et du lac du Bourget (France). Mémoire de master 2, ENS de Lyon, 160 p.

ONDE H. (1944). «Le lac d'Annecy et Le Thiou (étude hydrologique) ». Revue de géographie alpine, vol. $32, n^{\circ} 3$, p. 389-446.

ORR B.D., PICKENS J.B. (2003). "Public Access to Lake Superior and Attribute Values of Proximate Non-Shoreline Property". Journal of Great Lakes Research, vol. 29, n4, p. 616-629.

PERRET J.-P. (2009). « Droit spatialisé et espace juridiquement modelé ». In FOREST P. (dir.), Géographie du droit : épistémologie, développement et perspectives, Québec : Presses universitaires de Laval, p. 261-268.

PRIEUR L. (2012). « L'accès au rivage ». Revue juridique de l'environnement, n 5, p. 93-103.

ROLLO N., BARRAUD R., CHAUVEAU E. (2012). « Les enjeux sociospatiaux de la mise en place de la servitude de marchepied : l'exemple des rives de l'Erdre ». Les Cahiers nantais, vol. 2, p. 17-28.

SAJALOLI B. (1996). « Les zones humides : une nouvelle vitrine pour l'environnement ». Bulletin de l'Association de Géographes Français, vol. 73, n² 2, p. 132-144.

SONTAG K., ROUX F. (2013). « Loisirs sportifs et activités touristiques : aspects juridiques ». Journal of alpine research / Revue de géographie alpine, $\mathrm{n}^{\circ} 101-4$. 
TOMAS F. (2001). «L'espace public, un concept moribond ou en expansion? ». Géocarrefour, vol. 76, $\mathrm{n}^{\circ} 1, \mathrm{p} .75-84$.

TOUCHART L. (2000). Les lacs : origine et morphologie. Paris : L'Harmattan, coll. « Géotextes », 210 p.

ISBN 2-7384-9800-0

VERNEX J.-C. (1989). « Évolution des pratiques de loisirs et espace littoral lacustre. Une étude de cas : le lac d'Annecy (France) ». Le Globe, vol. 129, n 1, p. 37-47.

VERNEX J.-C. (1993). «L'invention des lieux touristiques ». Le Globe, vol. 133, n 1, p. 15-28.

VERNEX J.-C. (1996a). « Les lacs alpins, espaces saturés ? Pour une approche subjective de la

densité ». Méditerranée, vol. 84, n 3, p. 35-36.

VERNEX J.-C. (1996b). Histoire des bains : cent ans de baignades dans nos lacs. Léman, Annecy, Bourget.

Genève : Junod, $126 \mathrm{p}$.

VERNEX J.-C. (1998). « Qu'est-ce qu'un lac ? De l'imaginaire lacustre à l'aménagement ». Le Globe, vol. $138, \mathrm{n}^{\circ} 1, \mathrm{p} .7-16$.

VERNEX J.-C. (1985). « Paysage et société : l'exemple du littoral du lac d'Annecy ». Le Globe, vol. 125, $\mathrm{n}^{\circ} 1$, p. 273-287.

\section{NOTES}

1. Cet article est fondé sur le travail cartographique mené au cours d'une recherche plus globale (Nikolli, 2016), qui a abouti à un état des lieux social et juridique de la question de l'accès au lac (revue de la législation, revue de presse, analyse de dossiers d'enquête publique...) et à des entretiens semi-directifs avec les gestionnaires du lac et avec les associations citoyennes et environnementales locales. Ce travail incluait également une dimension comparative entre, d'une part, le lac d'Annecy (étudié en détail) et, d'autre part, le lac du Bourget et la rive française du Léman (étudiés de manière exploratoire). Ce travail qualitatif et sa dimension comparative n'étant pas centraux dans le propos, la méthodologie et les résultats n'en sont pas présentés en détail. Mais ces données complémentaires permettent d'éclairer les résultats du travail cartographique, notamment dans la dernière partie de l'article.

2. La zone de préemption est très peu utilisée par le département, dans la mesure où les espaces concernés ont été largement urbanisés depuis 1976, ce qui a vidé de son sens un mécanisme de préemption qui visait à acquérir et à protéger des espaces naturels. Ce droit de préemption est le plus souvent délégué au CELRL. Néanmoins, le Conseil départemental de Haute-Savoie travaille à une révision de la zone de préemption du lac d'Annecy.

3. Les neuf communes riveraines du lac sont soumises à la loi Littoral tandis que la loi Montagne s'applique dans 7 communes, partiellement à Annecy-le-Vieux et ne s'applique pas à Annecy.

4. Diaporama en ligne présentant les résultats de la cartographie des rives du lac par l'association Talloires Développement Durable : http://www.abcd-alpes.com/Multimedia/BERGES_2011/ SCO_0001/default.htm

5. Site de l'Association des Propriétaires Riverains du Lac d'Annecy (APRIL) : http://www.aprillacdannecy.com/site/

6. Le régime juridique des lacs domaniaux ne doit pas être assimilé à celui des littoraux maritimes. Mer et lacs relèvent de deux domaines publics différents, respectivement domaine public maritime (DPM) et domaine public fluvial (DPF). Au-delà des règles communes à tous les domaines publics (inaliénabilité et imprescriptibilité notamment), les différences sont nombreuses et ont des conséquences notables en matière d'accès à l'espace. 
La principale d'entre elles tient à la délimitation de ces deux domaines publics. Le domaine public maritime (DPM) intègre une partie terrestre : le «rivage de la mer» (jusqu'au niveau des plus hautes eaux ordinaires) et les « lais et relais de la mer », soient les dépôts alluviaux que la mer ne recouvre plus (relais), ou seulement lors du plus haut flot (lais) (CGPPP, art. L-5331-2). Le domaine public fluvial (DPF) est lui aussi délimité en référence aux plus hautes eaux ordinaires (art. L-2111-9 du CGPPP). Mais à la différence de la mer, le niveau des lacs varie peu, d'autant plus que le régime hydrologique naturel des grands lacs se trouve dans la plupart des cas régulé par des ouvrages de retenue. La limite terre/eau est donc beaucoup plus franche que dans le cas des littoraux maritimes et le DPF intègre beaucoup moins d'espaces périodiquement hors d'eau que le DPM. Dans les secteurs où les rives relèvent de la propriété privée, il n'y a donc pas systématiquement, au-devant des propriétés, de vaste grève qui relèverait du domaine public, et serait accessible à tous.

Au-delà de la limite du domaine public (maritime ou fluvial), les propriétés riveraines sont dans les deux cas grevées d'une servitude de passage. Mais ces deux servitudes sont nettement différentes, et ne doivent pas être confondues. La servitude de passage des piétons le long du littoral (SPPL), qui concerne les rivages maritimes, date de 1976 et constitue le socle d'un véritable droit d'accès au rivage (Prieur, 2012). Elle fait l'objet de conditions d'application spécifiques selon les configurations particulières qui peuvent se poser (falaises, propriétés privées trop proches de la limite du DPM...) (Couix, Le Roy, 1994). En revanche, la servitude de marchepied applicable aux cours d'eau et aux lacs domaniaux n'a été ouverte à tous les piétons qu'en 2006 (elle ne concernait auparavant que les services de l'État et les pêcheurs) et ne constitue encore qu'une disposition isolée et fort peu appliquée. La situation est encore plus spécifique sur les rives lacustres que sur les rives fluviales. Historiquement, les servitudes d'ordre public grevant les rives fluviales (halage notamment), n'ont que rarement été appliquées aux rives lacustres, pourtant intégrées dans le même droit (Larrieu, 1910). L'idée d'une servitude de passage fait donc beaucoup moins partie de la culture riveraine en bord de lac qu'en bord de mer ou de cours d'eau (exception faite du Léman, eu égard à sa situation frontalière et à l'existence historique d'un chemin du douanier).

Enfin, le droit du DPF a récemment connu des évolutions notables (Arzul, 2008), en particulier au regard des enjeux d'accès aux rives (possibilité de transfert de la propriété du DPF aux collectivités territoriales, renforcement de la servitude de marchepied...), quand le droit du DPM apparaît stabilisé depuis beaucoup plus longtemps.

7. Décret $n^{\circ} 72-72$ du 20 janvier 1972.

8. Elle a en revanche été fixée dès 1969 concernant le Léman, à l'occasion d'un contentieux entre l'État et des propriétaires riverains, et en 1999 pour le lac du Bourget, par arrêté préfectoral.

9. Le droit français distingue, au sein de la propriété publique, (i) le domaine public et (ii) le domaine privé des personnes publiques (à ne pas confondre avec la propriété privée). Le domaine public est soumis à des règles très particulières (inaliénabilité ; imprescriptibilité ; accès libre, gratuit et égal), regroupées depuis 2006 dans le CGPPP. Le domaine privé regroupe l'ensemble des biens publics qui ne font pas partie du domaine public, et n'obéit donc pas aux règles spécifiques du domaine public (Auby et al., 2016).

10. Cette première étape du travail cartographique est similaire à la méthode utilisée par Clotilde Buhot pour l'analyse de la propriété foncière sur les îles de Batz et de Bréhat, et détaillée dans son article (Buhot, 2005), à la différence près qu'il n'a pas été nécessaire de numériser et de digitaliser le parcellaire cadastral, directement récupéré sous forme de fichier SIG (BD Parcellaire, IGN).

11. $28 \%$ du linéaire de plages et $7 \%$ du linéaire total du lac.

12. Calculs SIG d'après les données cartographiques de l'association « Le lac pour tous » : http:// lelacpourtous.weebly.com/cartes.html 
13. Loi $n^{\circ}$ 2015-992 du 17 août 2015 relative à la transition énergétique. L'article 62 (codifié à l'art. L-2131-2 du CGPPP) confirme le principe de continuité de la servitude et n'autorise à s'éloigner de la rive qu'en cas « d'obstacle naturel ou patrimonial »; l'article 63 (art. L-2131-4 du CGPPP) autorise les communes, les établissements publics de coopération intercommunale, les départements, les syndicats mixtes et les associations d'usagers à saisir le préfet pour faire délimiter la servitude.

\section{RÉSUMÉS}

Avec le développement des pratiques récréatives, les espaces publics se renouvellent et sortent de la ville, brouillant les limites avec les espaces privés. L'article s'appuie sur le cas du lac d'Annecy (Haute-Savoie, France) et propose d'analyser l'espace public au croisement de la propriété foncière et de l'accès concret. Un travail cartographique met en lumière une relative déconnexion entre propriété et accès, et l'existence d'empiètements entre public et privé. À rebours des discours sur la privatisation des espaces publics, des cas de publicisation de l'espace privé sont mis en évidence.

As outdoor activities develop, public spaces are transformed and extend beyond cities, blurring the limits between public and private spaces. This paper relies on a case study of Lake Annecy (Haute-Savoie, France) and analyzes public space at the crossroads between private property and concrete access. Mapping both sets of data, we observe a relative disconnection between property and access, along with a mutual encroachment between public and private spaces. Contrary to dominant discourses about the privatization of public spaces, this research reveals certain cases of private spaces being rendered public.

Resumen : El desarrollo de las prácticas recreativas renuevan y alejan los espacios públicos de las ciudades, desdibujando sus límites con los espacios privados. A partir del estudio de caso del lago de Annecy (Alta Saboya, Francia), se propone un análisis de la noción de espacio público, y la dialéctica entre la propiedad privada de la tierra y el acceso público a las riberas. Mediante un análisis cartográfico se evidencian los problemas y múltiples superposiciones entre ambos. A contracorriente del tradicional discurso en torno a la privatización de los espacios públicos, este artículo presenta ejemplos de publicitación de los espacios privados.

\section{INDEX}

Keywords : right of access to the space, alpine lakes, privatization, land property, publicization Mots-clés : droit d'accès à l'espace, lacs alpins, privatisation, propriété foncière, publicisation Palabras claves : derecho de acceso al espacio, lagos alpinos, privatización, propiedad de la tierra, publicitación 


\section{AUTEUR}

\section{ALICE NIKOLLI}

Doctorante en géographie Laboratoire EDYTEM - Université Savoie Mont-Blanc 